PROCEEDINGS OF THE

AMERICAN MATHEMATICAL SOCIETY

Volume 140, Number 7, July 2012, Pages 2307-2318

S 0002-9939(2011)11100-6

Article electronically published on November 23, 2011

\title{
GRAPHS WITH AUTOMORPHISM GROUPS ADMITTING COMPOSITION FACTORS OF BOUNDED RANK
}

\author{
CHERYL E. PRAEGER, LASZLÓ PYBER, PABLO SPIGA, AND ENDRE SZABÓ
}

(Communicated by Jonathan I. Hall)

For the 60th birthday of L. Babai

\begin{abstract}
We prove a 1978 conjecture of Richard Weiss in the case of groups with composition factors of bounded rank. Namely, we prove that there exists a function $g: \mathbb{N} \times \mathbb{N} \rightarrow \mathbb{N}$ such that, for $\Gamma$ a connected $G$-vertex-transitive, $G$-locally primitive graph of valency at most $d$, if $G$ has no alternating groups of degree greater than $r$ as sections, then a vertex stabiliser in $G$ has size at most $g(r, d)$.
\end{abstract}

\section{INTRODUCTION}

A graph $\Gamma$ is said to be $G$-vertex-transitive if $G$ is a subgroup of Aut $(\Gamma)$ acting transitively on the vertex set $V \Gamma$ of $\Gamma$. We say that a $G$-vertex-transitive graph $\Gamma$ is $G$-locally primitive if the stabiliser $G_{\alpha}$ of the vertex $\alpha$ induces a primitive permutation group on the set $\Gamma(\alpha)$ of vertices adjacent to $\alpha$. In 1978 Richard Weiss 24] conjectured that for a finite connected $G$-vertex-transitive, $G$-locally primitive graph $\Gamma$, the size of $G_{\alpha}$ is bounded above by some function depending only on the valency of $\Gamma$. In spirit this conjecture is similar to the 1967 conjecture of Charles Sims [19] (stated in the graph theoretic context) for a $G$-vertex-primitive graph or digraph $\Gamma$, the size of the stabiliser of a vertex is bounded above by some function of the valency of $\Gamma$. In summary, in the conjecture of Weiss the graph $\Gamma$ is assumed to be locally primitive, connected, and vertex-transitive; in the conjecture of Sims the graph $\Gamma$ is assumed to be locally transitive and vertexprimitive (and hence connected). Despite the fact that the Sims Conjecture has been proved true in [8, the truth of the Weiss Conjecture is still unsettled and only partial results are known, much of them focussing on the "locally 2-transitive" case [10, 22, 23, 25, 26, 27, apart from the normal quotient reduction results in 9., 13.

Received by the editors December 16, 2010 and, in revised form, January 12, 2011 and February $28,2011$.

2000 Mathematics Subject Classification. Primary 20B25.

Key words and phrases. Weiss conjecture, normal quotients, quasiprimitive groups, almost simple groups.

The first author is supported by the ARC Federation Fellowship Project FF0776186.

The second author is supported in part by OTKA 78439 and 72523.

The third author is supported by the University of Western Australia as part of the Federation Fellowship project.

The fourth author is supported in part by OTKA 81203 and 72523.

(C)2011 American Mathematical Society 
In this paper we discuss the Weiss Conjecture and prove it for groups with composition factors of bounded rank.

Definition 1. Define $\operatorname{BCP}(r)$ to be the class of finite groups $G$ such that there is no section $H / K$ of $G$, where $K<H \leq G$ and $K$ is normal in $H$, isomorphic to the alternating group $\operatorname{Alt}(r+1)$.

The class of $\mathrm{BCP}(r)$-groups was first considered by Babai, Cameron and Pálfy 1 . They showed that primitive $\operatorname{BCP}(r)$-groups of degree $n$ have order at most $n^{f(r)}$. This result is an essential ingredient of many polynomial time algorithms for permutation groups related to the graph isomorphism problem 11. The $\mathrm{BCP}(r)$-groups also play a very important role in the theory of subgroup growth of residually finite groups (see [12). This paper uncovers a new application of this class of groups to the Weiss Conjecture.

We note that a group $G \in \mathrm{BCP}(r)$ does not have as a composition factor a simple group of Lie type of rank at least $r+1$, as such simple groups have $\operatorname{Alt}(r+1)$ as a section. On the other hand, if $G$ has no simple groups of Lie type (resp. alternating groups) of rank (resp. degree) at least $k$ as composition factors, then $G \in \mathrm{BCP}(r)$ for some $r \leq C k$. This justifies our reference to these groups as having composition factors of bounded rank.

Theorem 2. There exists a function $g: \mathbb{N} \times \mathbb{N} \rightarrow \mathbb{N}$ such that for $\Gamma$ a connected $G$ vertex-transitive, $G$-locally primitive graph of valency at most d, if $G$ is a $\mathrm{BCP}(r)$ group, then a vertex stabiliser in $G$ has size at most $g(r, d)$.

Remark 3. In light of this theorem the Weiss Conjecture asks whether the function $g$ can be chosen so as not to depend on $r$.

Let $\Gamma$ be a connected $G$-vertex-transitive graph of valency at most $d$. If $G$ has a normal subgroup $K$ with at least three orbits on $V \Gamma$, then the group $H=G / K$ is called an intransitive head of $G$. We write $\alpha^{K}$ for the $K$-orbit of the vertex $\alpha$ of $\Gamma$. The normal quotient $\Gamma_{K}$ is the graph whose vertices are the orbits of $K$ on $V \Gamma$, with an edge between two distinct vertices $\alpha^{K}$ and $\beta^{K}$ in $\Gamma_{K}$, if and only if there is an edge of $\Gamma$ between $\alpha^{\prime}$ and $\beta^{\prime}$ for some $\alpha^{\prime} \in \alpha^{K}$ and some $\beta^{\prime} \in \beta^{K}$. It was proved in [13, Section 1] that $\Gamma_{K}$ is an $H$-vertex-transitive graph of valency at most $d$. Furthermore, if $\Gamma$ is $G$-locally primitive, then $\Gamma_{K}$ is $H$-locally primitive and the vertex stabilisers for $H$ on $\Gamma_{K}$ and for $G$ on $\Gamma$ are isomorphic groups. Thus for proving Theorem 2 it is sufficient to consider the case where there is no non-trivial normal quotient reduction. The groups $G$ without non-identity normal subgroups $K$ with at least three orbits on $V \Gamma$ (and hence admitting no reduction) are called quasiprimitive (if every non-identity normal subgroup of $G$ is transitive) and biquasiprimitive (if $G$ is not quasiprimitive and every non-identity normal subgroup of $G$ has at most two orbits).

In [9] an analysis of $G$-locally primitive graphs with $G$ quasiprimitive on vertices was undertaken, considering separately each of the eight types of quasiprimitive groups according to the quasiprimitive groups subdivision described in [14]. For six of the eight quasiprimitive types it was proved that $\left|G_{\alpha}\right|$ is bounded above by an explicit function of the valency, reducing the problem of proving the Weiss Conjecture for quasiprimitive groups $G$ to the almost simple and product action types AS and PA (9, Section 2]). The PA type was also examined in [9, Proposition 2.2], but unfortunately the proof contains an error. (We explain the mistake in Remark 9]) 
In this paper we actually prove a more general result from which, in the light of the comment above, Theorem 2 follows immediately.

Theorem 4. There exists a function $g: \mathbb{N} \times \mathbb{N} \rightarrow \mathbb{N}$ such that for $\Gamma$ a connected $G$-vertex-transitive, $G$-locally primitive graph of valency at most $d$, if $G$ has an intransitive head that is a $\mathrm{BCP}(r)$-group, then a vertex stabiliser in $G$ has size at most $g(r, d)$.

The proof of this result makes use of new results in two separate areas. First we apply new results of Praeger, Spiga and Verret [16] which reduce the proof of Theorem 4 to consideration of the case of finite simple groups $G$. Then we apply the new results of Pyber, Szabó [17] and Breuillard, Green, Tao 44 on growth in simple groups.

We mention that the results in [16] are for vertex-transitive graphs, and in this context we prove the following more general version of Theorem 4 .

Theorem 5. There exists a function $g: \mathbb{N} \times \mathbb{N} \rightarrow \mathbb{N}$ such that for $\Gamma$ a connected $G$-vertex-transitive graph of valency at most $d$, if $G$ has a $\operatorname{BCP}(r)$-group $G / K$ as an intransitive head and $G / K$ is quasiprimitive or biquasiprimitive on $\mathrm{V}_{K}$, then for a vertex $\alpha$ we have $\left|G_{\alpha} / K_{\alpha}\right| \leq g(r, d)$.

If the graph $\Gamma$ is $G$-locally primitive, then by [13. Section 1] the subgroup $K_{\alpha}$ is trivial (and so Theorem 4 follows immediately from Theorem 5). As we noted in Remark 3, the Weiss Conjecture would assert that in this case the function $g$ can be chosen so as not to depend on $r$. More generally we ask:

Question 6. What is the weakest local assumption that guarantees a bound on the size of $G_{\alpha} / K_{\alpha}$ in terms of the valency $d$ alone?

In Section 3 we give examples which show that some local assumption is needed even in the case when $G$ is quasiprimitive. Namely, we construct connected $G$-arctransitive graphs $\Gamma$ of valency $2 r$ such that $G \cong \operatorname{Sym}((m+1) r-1)$ and $G_{\alpha} \cong$ $\operatorname{Alt}(r)^{m-2} \times \operatorname{Alt}(r-1)$ for all $m \equiv 3 \bmod 4$ and $r \geq 3$.

These examples also yield generating sets $A$ of size $2(r !)^{m-1}$ in $\operatorname{Sym}((m+1) r-1)$ such that $\left|A^{3}\right| \leq 4 r^{2}|A|$. This shows that the analogues of the results obtained in [17] and [4] for bounded rank families of simple groups of Lie type do not hold for the family of finite symmetric groups.

It would be interesting to know whether there exist families of $G$-arc-transitive graphs of fixed valency with unbounded vertex stabilisers (where each $G$ is isomorphic to some alternating or symmetric group) which are essentially different from the ones mentioned above. Motivated by the known examples we ask the following:

Question 7. Let $\Gamma$ be a connected $G$-vertex-transitive graph or digraph. Is it true that the exponent of $G_{\alpha}$ is bounded in terms of the valency $d$ ?

A positive answer, even in the case when $G$ is non-abelian simple, would be of great interest.

Theorem 5 raises another question:

Question 8. What is the weakest local assumption that guarantees a bound on the size of $K_{\alpha}$ in terms of the valency, for an intransitive normal subgroup $K$ ?

There are infinite families of vertex-transitive (and arc-transitive) graphs of fixed valency $d$ with intransitive heads $G / K$ such that $K_{\alpha}$ is unbounded. For example, 
in the case of wreath graphs $C_{n}\left[\bar{K}_{d / 2}\right]$ of even valency $d$ (that is, the lexicographic product of a cycle of length $n$ with an edgeless graph on $d / 2$ vertices), we have $G=\operatorname{Sym}(d / 2)$ wr $D_{2 n}, K=\operatorname{Sym}(d / 2)^{n}$ and $K_{\alpha}=\operatorname{Sym}(d / 2-1) \times \operatorname{Sym}(d / 2)^{n-1}$.

Remark 9. In 9 the $G$-vertex-quasiprimitive, $G$-locally primitive graphs were analysed and an attempt was made to reduce the proof of the Weiss Conjecture in this case to the situation where $G$ is an almost simple group. This was only partially successful: when working on this paper a subtle mistake in the handling of the product action case (quasiprimitive groups of type PA) was discovered. The result 9 , Proposition 2.2] cannot be recovered, as we were able to construct a counterexample; see Example 16. This example demonstrates that there is no natural reduction to the case of $G$ almost simple. A completely new combinatorial approach was needed, developed in [16, to enable powerful new results from [17] and [4 to be applied.

Example 16 gives a connected graph $\Gamma$ of valency 9 and a group $G \leq \operatorname{Aut}(\Gamma)$ with $G$ quasiprimitive on vertices of product action type PA and a vertex stabiliser inducing $\operatorname{Sym}(3)$ wr Sym(2) in its primitive product action on the vertex neighbourhood. There is a naturally defined associated $H$-arc-transitive graph of valency 9 where $H$ is almost simple with socle $T$, with $T$ the simple direct factor of the socle of $G$. The (incorrect) proof of [9, Proposition 2.2] asserts that this graph is $H$-locally primitive. However, for this graph the local action induced by a vertex stabiliser in $H$ is $\operatorname{Sym}(3) \times \operatorname{Sym}(3)$ (having two intransitive normal subgroups $\operatorname{Sym}(3)$ ).

\section{Proofs of the theOrems}

We start by deriving Theorems 2 and 4 from Theorem 5

Proof of Theorems 2 and 4 from Theorem 5 . Let $g$ be the function in the statement of Theorem 5 Assume that $\Gamma$ is a connected $G$-vertex-transitive and $G$-locally primitive graph of valency at most $d$ and that $G$ has an intransitive head $G / K^{\prime}$ that is a $\operatorname{BCP}(r)$-group. (For Theorem 2 take $K^{\prime}=1$.) Choose $K$ maximal such that $K$ has at least three orbits on the vertices of $\Gamma$ with $K^{\prime} \subseteq K$. We conclude that the action of $G / K$ on the set of $K$-orbits is faithful and is either quasiprimitive or biquasiprimitive. Since $G / K^{\prime}$ is a $\mathrm{BCP}(r)$-group and $K^{\prime} \subseteq K$, we have that $G / K$ is a $\mathrm{BCP}(r)$-group. Let $\alpha$ be a vertex. By Theorem $5,\left|G_{\alpha}\right| /\left|K_{\alpha}\right| \leq g(r, d)$. Since $K_{\alpha}$ is normal in $G_{\alpha}$ and since $G_{\alpha}$ induces a primitive action on the set $\Gamma(\alpha)$ of neighbours of $\alpha$, either (i) $K_{\alpha}$ is transitive on $\Gamma(\alpha)$ or (ii) $K_{\alpha}$ fixes $\Gamma(\alpha)$ pointwise. We now use the fact that $\Gamma$ is connected. In case (i), since $G$ is vertex-transitive, $K_{\beta}$ is transitive on $\Gamma(\beta)$ for all vertices $\beta$, and it follows from connectivity that $K$ is edge-transitive and so has at most two orbits on vertices, contradicting the assumption that $G / K$ is an intransitive head. In case (ii) by connectivity, $K_{\alpha}$ fixes every vertex of $\Gamma$, and so $K_{\alpha}=1$. Hence $\left|G_{\alpha}\right| \leq g(r, d)$.

Before embarking on the proof of Theorem 5 we recall the definition of a coset graph and some elementary results.

Definition 10. Let $G$ be a group, $H$ a subgroup of $G$ and $A$ a subset of $G$. The coset digraph $\operatorname{Cos}(G, H, A)$ is the digraph with vertex set the right cosets of $H$ in $G$ and with arcs the ordered pairs $(H x, H y)$ such that $H y x^{-1} H \subseteq H A H$ (where $H A H=\{h s k \mid h, k \in H, a \in A\}$ ). Since $\operatorname{Cos}(G, H, A)=\operatorname{Cos}(G, H, H A H)$, 
replacing $A$ by $H A H$, we may assume that $A$ is a union of $H$-double cosets; that is, $A$ is a disjoint union $\bigcup_{s \in S} H s H$ for some subset $S$ of $G$.

It is immediate to check that $\operatorname{Cos}(G, H, A)$ is undirected if and only if $A=A^{-1}$ and that $\operatorname{Cos}(G, H, A)$ is connected if and only if $G=\langle A\rangle$. Also the action of $G$ by right multiplication of $G / H$ induces a vertex-transitive automorphism group of $\operatorname{Cos}(G, H, A)$.

It was proved by Sabidussi $[18$ that every $G$-vertex-transitive graph $\Gamma$ is isomorphic to some coset graph of $G$. More precisely, we have the following well-known result.

Proposition 11. Let $\Gamma$ be a $G$-vertex-transitive graph and $\alpha$ a vertex of $\Gamma$. Then there exists a union $A$ of $G_{\alpha}$-double cosets such that $\Gamma \cong \operatorname{Cos}\left(G, G_{\alpha}, A\right)$ and with the action of $G$ on $V \Gamma$ equivalent to the action of $G$ by right multiplication on the right cosets of $G_{\alpha}$ in $G$.

In the proof of Theorem 5 we will use two new results (which we report below), one combinatorial [16] (see Theorem 13) and the other group theoretic 17] (see Theorem 14). For stating Theorem 13 we need the following definition (see [14] and [15, Theorem 1.1]). Also we denote the set of functions $\mathbb{N} \rightarrow \mathbb{N}$ by $\operatorname{Func}(\mathbb{N})$.

Definition 12. If $G$ is a quasiprimitive or biquasiprimitive permutation group with socle $T^{\ell}$ with $T$ simple, then we call $T$ the socle factor of $G$.

Theorem 13 (Theorems 4 and 5 in [16]). There exists a function $h: \mathbb{N} \rightarrow \mathbb{N}$ such that for $\Gamma$ a connected $G$-vertex-transitive graph of valency at most $d$ and $\alpha$ a vertex of $\Gamma$, if $G$ is quasiprimitive or biquasiprimitive on vertices with socle factor $T$, then either

(1) $\left|G_{\alpha}\right| \leq h(d)$ or

(2) $\Gamma$ and $G$ uniquely determine two (possibly isomorphic) connected $T$-vertextransitive graphs $\Lambda_{1}$ and $\Lambda_{2}$ of valency at most $d(d-1)$. Also, there is a function $p: \mathbb{N} \times \operatorname{Func}(\mathbb{N}) \times \operatorname{Func}(\mathbb{N}) \rightarrow \mathbb{N}$ such that if for each $i=1,2$, $\left|T_{\lambda_{i}}\right| \leq g_{i}(d(d-1))$ for $\lambda_{i} \in V \Lambda_{i}$ and for some functions $g_{i}: \mathbb{N} \rightarrow \mathbb{N}$, then $\left|G_{\alpha}\right| \leq p\left(d, g_{1}, g_{2}\right)$.

The following result already has many applications to the construction of families of expander graphs and in number theory; see e.g. 2, 3, 5, 6]. Here we give a rather different kind of application.

Theorem 14 (Theorem 3 in 17; see also Theorem 2.3 in 44). Let $T$ be a simple group of Lie type of rank $r$ and $A$ a generating set of $T$. Then either $T=A^{3}$ or $|A|^{1+\varepsilon(r)} \leq c(r)\left|A^{3}\right|$ with positive constants $c(r)$ and $\varepsilon(r)$ depending only on the Lie rank $r$ of the simple group $T$.

In Lemma 15 we derive from Theorem 14 the proof of Theorem 5 in the preliminary case that the group $T$ of automorphisms of the graph $\Gamma$ is a $\mathrm{BCP}(r)$-group with $T$ simple.

Lemma 15. There exists a function $f: \mathbb{N} \times \mathbb{N} \rightarrow \mathbb{N}$ such that for $\Gamma$ a connected $T$ vertex-transitive graph of valency at most $d$, if $T$ is a $\mathrm{BCP}(r)$-group with $T$ simple, then a vertex stabiliser in $T$ has size at most $f(r, d)$.

Proof. By Proposition 11, we may identify $\Gamma$ with $\operatorname{Cos}\left(T, T_{\alpha}, A\right)$ and the action of $T$ on $\Gamma$ with the action of $T$ by right multiplication on the right cosets of $T_{\alpha}$ in $T$, for some union $A$ of $T_{\alpha}$-double cosets. 
Assume that $T$ is abelian or a sporadic simple group or $\operatorname{Alt}(n)$ for $n \leq r$. Clearly $\left|T_{\alpha}\right| \leq \max \{r !,|M|\}$, where $M$ is the Monster sporadic simple group.

Assume that $T$ is a simple group of Lie type. As $T$ is a $\operatorname{BCP}(r)$-group, then, as noted in the introduction, $T$ has Lie rank at most $r$. Since $\Gamma$ has valency $d_{0} \leq d$ and the neighbours of the vertex $T_{\alpha}$ are the $T_{\alpha}$-right cosets contained in $A$, we have $|A|=d_{0}\left|T_{\alpha}\right|$. We claim that $\left|A^{3}\right| \leq d_{0}^{3}\left|T_{\alpha}\right|$. Let $x$ be in $A^{3}$ and write $x=a_{1} a_{2} a_{3}$ with $a_{1}, a_{2}$ and $a_{3}$ in $A$. By definition of the coset graph,

$$
\left(T_{\alpha}, T_{\alpha} a_{3}, T_{\alpha} a_{2} a_{3}, T_{\alpha} a_{1} a_{2} a_{3}\right)
$$

is a path of length 3 from $T_{\alpha}$ to $T_{\alpha} x$ in $\Gamma$. Thus every vertex $T_{\alpha} x$ of $\Gamma$ with $x$ in $A^{3}$ is at distance at most 3 from $T_{\alpha}$. Since $\Gamma$ has valency $d_{0}$, the number of vertices at distance at most 3 from $\alpha$ is at most $1+d_{0}+d_{0}\left(d_{0}-1\right)+d_{0}\left(d_{0}-1\right)^{2} \leq d_{0}^{3}$ for $d_{0} \geq 2$. Since $A$ is a union of right $T_{\alpha}$-cosets, we obtain $\left|A^{3}\right| \leq d_{0}^{3}\left|T_{\alpha}\right|$, proving the claim.

Since $\Gamma$ is a connected undirected graph, we have $T=\langle A\rangle$. From Theorem 14, we obtain that either $T=A^{3}$ or there exist positive constants $c(r)$ and $\varepsilon(r)$, depending only on the Lie rank $r$ of the simple group $T$, such that $|A|^{1+\varepsilon(r)} \leq c(r)\left|A^{3}\right|$. If $T=A^{3}$, then

$$
|V \Gamma|=\left|T: T_{\alpha}\right|=\frac{\left|A^{3}\right|}{\left|T_{\alpha}\right|} \leq d_{0}^{3} \leq d^{3},
$$

and hence the number of vertices of $\Gamma$ is bounded by a function of $d$. In particular, $\left|T_{\alpha}\right| \leq d^{3}$ !. If $|A|^{1+\varepsilon(r)} \leq c(r)\left|A^{3}\right|$, then

$$
\left(d_{0}\left|T_{\alpha}\right|\right)^{1+\varepsilon(r)}=|A|^{1+\varepsilon(r)} \leq c(r)\left|A^{3}\right| \leq c(r) d_{0}^{3}\left|T_{\alpha}\right|,
$$

which yields

$$
\left|T_{\alpha}\right| \leq\left(c(r) d_{0}^{2-\varepsilon(r)}\right)^{1 / \varepsilon(r)} \leq\left(c(r) d^{2-\varepsilon(r)}\right)^{1 / \varepsilon(r)},
$$

and thus in all cases $\left|T_{\alpha}\right| \leq f(r, d)$ where

$$
f(r, d)=\max \left\{r !,|M|, d^{3} !,\left(c(r) d^{2-\varepsilon(r)}\right)^{1 / \varepsilon(r)}\right\} .
$$

Finally we are ready to prove Theorem 5 .

Proof of Theorem 5. Let $h$ and $p$ be the functions in the statement of Theorem 13 and $f$ the function in the statement of Lemma 15, Define $g: \mathbb{N} \times \mathbb{N} \rightarrow \mathbb{N}$ by $g(r, d)=\max \{h(d), p(d, f(r, d(d-1)), f(r, d(d-1)))\}$.

Assume that $\Gamma$ is a connected $G$-vertex-transitive graph of valency at most $d$, that $G$ has an intransitive head $G / K$ that is a $\operatorname{BCP}(r)$-group, and that $G / K$ is quasiprimitive or biquasiprimitive on $\mathrm{V} \Gamma_{K}$. Let $\alpha$ be a vertex of $\Gamma$. We consider the action of $G / K$ on the normal quotient graph $\Gamma_{K}$. Since $\Gamma$ has valency at most $d$, we obtain that $\Gamma_{K}$ has valency at most $d$. The socle of $G / K$ is $T^{\ell}$ for some simple group $T$ and integer $\ell$ (by 14 and [15. Theorem 1.1]). Note that the stabiliser in $G$ of the vertex $B=\alpha^{K}$ of $\Gamma_{K}$ is $G_{B}=K G_{\alpha}$ and that $\left|K G_{\alpha} / K\right|$ (the size of the stabiliser in the action of $G / K$ on $\left.\Gamma_{K}\right)$ is equal to $\left|G_{\alpha}\right| /\left|K_{\alpha}\right|$.

Now we apply Theorem 13 to $\Gamma_{K}$ and $G / K$. If Part (1) of Theorem 13 holds, then $\left|G_{\alpha}\right| /\left|K_{\alpha}\right| \leq h(d) \leq g(r, d)$. Hence we may assume that Part (2) of Theorem 13 holds for $G / K$ and $\Gamma_{K}$. This gives two (possibly isomorphic) graphs $\Lambda_{i}$, each of valency at most $d(d-1)$, admitting $T$ acting vertex-transitively. Let $\lambda_{i}$ be a vertex of $\Lambda_{i}$ for $i=1,2$. 
Since $G / K$ is a $\operatorname{BCP}(r)$-group, so is $T$. Now, for each $i=1,2$, we apply Lemma 15 to $\Lambda_{i}$ and $T$, and we obtain $\left|T_{\lambda_{i}}\right| \leq f(r, d(d-1))$. Hence from Theorem $13(2)$, we get $\left|G_{\alpha}\right| /\left|K_{\alpha}\right| \leq p(d, f(r, d(d-1)), f(r, d(d-1))) \leq g(r, d)$, and the theorem is proved.

We conclude this section by giving the example described in Remark 9 ,

Example 16. The graph $\Gamma$ will be a connected $G$-vertex-transitive, $G$-locally primitive graph of valency 9 , where $G \leq \operatorname{Sym}(10)$ wr $\operatorname{Sym}(2)$ is quasiprimitive of type PA with socle Alt $(10)^{2}$. The naturally defined associated Sym(10)-arc-transitive graph will have valency 9 , and the local action induced by $\operatorname{Sym}(10)$ is the product action of $\operatorname{Sym}(3) \times \operatorname{Sym}(3)$ which is imprimitive (having two intransitive normal subgroups $\operatorname{Sym}(3))$.

Let $H=\operatorname{Sym}(10), x=(1,2,3)(4,5,6)(7,8,9), y=(1,4,7)(2,5,8)(3,6,9), z=$ $(2,3)(5,6)(8,9), t=(4,7)(5,8)(6,9)$ and $\iota=(1,10)$. Write $K=\langle x, y, z, t\rangle$. Clearly, $K=\langle x, z\rangle \times\langle y, t\rangle \cong \operatorname{Sym}(3) \times \operatorname{Sym}(3)$. Let $\Delta$ be the $H$-set $H / K$ and $\Lambda$ the coset graph $\operatorname{Cos}(H, K, K \iota K)$. Since $\iota$ is an involution, $\Lambda$ is undirected. Also, as $K \cap K^{\iota}=\langle z, t\rangle$ and $|K:\langle z, t\rangle|=9$, the graph $\Lambda$ has valency 9 and the local action is the natural product action of $\operatorname{Sym}(3) \times \operatorname{Sym}(3)$ of degree 9 . Furthermore, it is easy to check that $H=\langle K, \iota\rangle$, and hence the graph $\Lambda$ is a connected $H$-arc-transitive vertex-quasiprimitive graph.

Let $W$ be the wreath product $H$ wr $\operatorname{Sym}(2)=(H \times H) \rtimes\langle\pi\rangle$, where $\pi^{2}=1$ and $\left(h_{1}, h_{2}\right)^{\pi}=\left(h_{2}, h_{1}\right)$ for $h_{1}, h_{2} \in H$. Let $T$ be the socle of $H$ and $N=T^{2}$ the socle of $W$. Consider $G=N \rtimes\langle\pi,(\iota, \iota)\rangle$. The group $N\langle(\iota, \iota)\rangle=G \cap(H \times H)$ is the subgroup of index 2 of $H^{2}$ normalised by $\pi$. Note that each of $\pi,(\iota, \iota)$ has order 2 and $(\iota, \iota)^{\pi}=(\iota, \iota)$. So $G / N$ is an elementary abelian group of order 4 . Also, the projection of $N_{G}(T \times 1)=N\langle(\iota, \iota)\rangle$ onto the first coordinate of $H^{2}$ is the whole of $H$. Consider the subgroup $L=\langle(x, y),(y, x),(z, t),(t, z), \pi\rangle$ of $G$. Note that $\langle(x, y),(y, x),(t, z),(z, t)\rangle$ is a diagonal subgroup of $K \times K$ normalised by $\pi$. Furthermore, $\langle(x, y),(z, t)\rangle^{\pi}=\left\langle(x, y)^{\pi},(z, t)^{\pi}\right\rangle=\langle(y, x),(t, z)\rangle \cong \operatorname{Sym}(3)$ and also $\langle(x, y),(z, t)\rangle$ and $\langle(y, x),(t, z)\rangle$ commute. Therefore we have that $|L|=72$ and $L$ is isomorphic to $\operatorname{Sym}(3) \mathrm{wr} \operatorname{Sym}(2)$.

Let $\Omega$ be the $G$-set $G / L$. As $z, t \in \operatorname{Sym}(10) \backslash \operatorname{Alt}(10)$ and $(z, t) \in L$, we have $N\langle(\iota, \iota)\rangle=N\langle(z, t)\rangle \subseteq N L \subseteq G$. Also, since $\pi \in L$, we obtain $G=N L$. Clearly $N \cap L=\langle(x, y),(y, x),(z t, t z)\rangle$ has order 18. In particular, $N$ is the unique minimal normal subgroup of $G$ and is transitive on $\Omega$ since $G=N L$. Thus $G$ is quasiprimitive on $\Omega$. Since $N \cap L$ projects to proper non-trivial subgroups of $T$, the group $G$ has quasiprimitive type PA (see [14]).

Note that the group $(K \cap T) \times(K \cap T)=\langle(x, 1),(y, 1),(z t, 1)\rangle \times\langle(1, x),(1, y),(1, z t)\rangle$ is normalised by $(z, t)$ and $\pi$, and hence by $L$. Consider $L^{*}=((K \cap T) \times(K \cap T)) L$ and $\Sigma$ the system of imprimitivity of $\Omega$ corresponding to the overgroup $L^{*}$ of $L$. As $z, t \in \operatorname{Sym}(10) \backslash \operatorname{Alt}(10)$, we have $L^{*}=((K \cap T) \times(K \cap T))\langle(z, t), \pi\rangle$ and $(K \cap T) \times(K \cap T)$ is normal in $L^{*}$. Since $(K \cap T) \times(K \cap T)$ has order $18^{2}=324$ and $\left|L^{*}:((K \cap T) \times(K \cap T))\right|=4$, we have $\left|L^{*}\right|=4 \cdot 324=1296$.

Since $L^{*} \cap N=(K \cap T) \times(K \cap T)$, we have that the $N$-space $\Sigma$ is permutation equivalent to the $N$-space $D^{2}$, where $D=T /(K \cap T)$. As the action of $T$ on $D$ is equivalent to its action on $\Delta$, we obtain that the $N$-space $\Sigma$ is equivalent to $\Delta^{2}$ with $N$ acting in product action. Finally, since $\Sigma$ is $G$-invariant, the action of $G$ on $\Sigma$ is equivalent to its product action on $\Delta^{2}$. 
Let $\Gamma$ be the coset graph $\operatorname{Cos}(G, L, L(t z \iota, \iota) L)$. Denote by $\alpha$ the vertex $L$ of $\Gamma$ and by $\beta$ the vertex $L(t z \iota, \iota)$ of $\Gamma$. Since the involutions $z, t$ and $\iota$ of $H$ are pairwise commuting, the element $(t z \iota, \iota)$ is an involution of $G$ interchanging $\alpha$ and $\beta$, and hence $\Gamma$ is undirected. Furthermore, $(z, t)^{(z t \iota, \iota)}=(t, z) \in L,(t, z)^{(z t \iota, \iota)}=$ $(t, z) \in L$ and $\pi^{(z t \iota, \iota)}=(z t \iota, \iota) \pi(z t \iota, \iota)=(z t \iota, \iota)(\iota, z t \iota) \pi=(z t, z t) \pi \in L$. Hence $\left|L: L^{(z t \iota, \iota)}\right| \leq 9$. A similar computation with $(x, y)$ and $(y, x)$ shows that $\mid L \cap$ $L^{(z t \iota, \iota)} \mid \geq 9$, and hence $L \cap L^{(z t \iota, \iota)}=\langle(t, z),(z, t), \pi\rangle$. This gives that $\left|G_{\alpha}: G_{\alpha, \beta}\right|=$ $\left|L: L \cap L^{(z t \iota, \iota)}\right|=9$, and so $\Gamma$ has valency 9. Moreover, as $L \cap L^{(z t \iota, \iota)}$ is a Sylow 2-subgroup of $L$ and any two distinct Sylow 2-subgroups of $L$ generate the whole of $L$, we obtain that the action of $G_{\alpha}$ on $\Gamma(\alpha)$ is primitive. Then $\Gamma$ is $G$-locally primitive with the claimed local action.

It is easy to show with the invaluable help of Magma [7] that $G=\langle L,(z t \iota, \iota)\rangle$, from which it follows that $\Gamma$ is connected.

\section{THE MAIN EXAMPLES}

In this section we construct connected $G$-arc-transitive graphs for which $G_{\alpha}$ can be arbitrarily large compared to the valency. Recall that by the ThompsonWielandt theorem [21, if $G$ is a primitive group and $d$ is the size of a suborbit, then for some prime $p$ the size of $G_{\alpha} / O_{p}\left(G_{\alpha}\right)$ is bounded by some function of $d$ (this result is the starting point of the proof of the Sims Conjecture [8]). The examples given below show that even the analogue of the Thompson-Wielandt theorem fails for quasiprimitive groups.

As noted in the introduction, these examples also yield exponentially large generating sets of symmetric groups with very small growth.

Let $m$ be an integer with $m \equiv 3 \bmod 4, r \geq 3$ and $\Omega=\{1, \ldots, r-1+m r\}$. Let $\mathcal{P}=\left\{X_{0}, \ldots, X_{m}\right\}$ be the partition of $\Omega$ defined by

$$
\begin{aligned}
X_{j} & =\{1+j r, \ldots, r+j r\}, \quad \text { for } j=0, \ldots, m-1, \\
X_{m} & =\{1+m r, \ldots, r-1+m r\} .
\end{aligned}
$$

In particular, for $j \in\{0, \ldots, m-1\}$, we have $\left|X_{j}\right|=r$ and $\left|X_{m}\right|=r-1$. For each $j \in\{0, \ldots, m\}$, write $\operatorname{Alt}\left(X_{j}\right)$ for the alternating group on $X_{j}$ fixing point-wise $\Omega \backslash X_{j}$. Note that for $i, j \in\{0, \ldots, m\}$ with $i \neq j$ we have that $\operatorname{Alt}\left(X_{i}\right)$ centralises $\operatorname{Alt}\left(X_{j}\right)$. Set

$$
H_{0}=\prod_{j=0}^{m} \operatorname{Alt}\left(X_{j}\right) \cong \operatorname{Alt}(r)^{m} \times \operatorname{Alt}(r-1) .
$$

Define the following permutation of $\Omega$ :

(3) $h: \begin{cases}z+l r & \mapsto z+(m-l-1) r \\ z+m r & \mapsto z+m r\end{cases}$

for $1 \leq z \leq r, 0 \leq l \leq m-1$, for $1 \leq z \leq r-1$.

Clearly $h$ is an involution of $\operatorname{Sym}(\Omega)$ centralising $\operatorname{Alt}\left(X_{(m-1) / 2}\right)$ and $\operatorname{Alt}\left(X_{m}\right)$. Furthermore, for each $j \in\{0, \ldots, m-1\}$, we have $X_{j}^{h}=X_{m-j-1}$, and hence $\operatorname{Alt}\left(X_{j}\right)^{h}=\operatorname{Alt}\left(X_{m-j-1}\right)$. Therefore $h$ normalises $H_{0}$. Set

$$
H=\left\langle H_{0}, h\right\rangle \cong\left(\operatorname{Alt}(r)^{m} \rtimes C_{2}\right) \times \operatorname{Alt}(r-1) .
$$


Define the following permutation of $\Omega$ :

(5) $a:\left\{\begin{array}{llll}z & \mapsto z+m r & & \text { for } 1 \leq z \leq r-1, \\ r & & \mapsto r, & \\ z+l r & \mapsto z+(l+1) r & \text { for } 1 \leq z \leq r, 1 \leq l \leq m-1, l \text { odd } \\ z+l r & \mapsto z+(l-1) r & & \text { for } 1 \leq z \leq r, 1 \leq l \leq m-1, l \text { even, } \\ z+m r & \mapsto z & & \text { for } 1 \leq z \leq r-1 .\end{array}\right.$

Write $X_{0}^{\prime}=X_{0} \backslash\{r\}$. Clearly $a$ is an involution of $\operatorname{Sym}(\Omega)$ with

$$
\begin{aligned}
& X_{j}^{a}=X_{j+1} \quad \text { and } \quad X_{j+1}^{a}=X_{j}, \quad \text { for } 1 \leq j \leq m-1, j \text { odd } \\
& X_{j}^{a}=X_{j-1} \quad \text { and } \quad X_{j-1}^{a}=X_{j},
\end{aligned}
$$

In particular, $a$ normalises the subgroup $\operatorname{Alt}\left(X_{0}^{\prime}\right) \times \operatorname{Alt}\left(X_{1}\right) \times \cdots \times \operatorname{Alt}\left(X_{m}\right)$ of $H$.

Given a $G$-arc-transitive graph $\Gamma$ and $v$ a vertex of $\Gamma$, we write $G_{1}(v)$ for the point-wise stabiliser of the neighbourhood $\Gamma(v)$ of $v$.

Theorem 17. Let $m, r, \Omega, H$ and $a$ be as above, $G=\operatorname{Sym}(\Omega)$ and $\Gamma=$ $\operatorname{Cos}(G, H, H a H)$. Then $\Gamma$ is a connected $G$-arc-transitive graph of valency $2 r$ and for a vertex $v, G_{v}^{\Gamma(v)} \cong \operatorname{Alt}(r) \mathrm{wr} C_{2}$ (in its imprimitive action of degree $2 r$ ) and $G_{1}(v) \cong \operatorname{Alt}(r)^{m-2} \times \operatorname{Alt}(r-1)$.

For the generating set $H a H$ of $\operatorname{Sym}(\Omega)$ we have $\left|(H a H)^{3}\right| \leq 4 r^{2}|H a H|$.

Proof. We prove three claims from which the theorem will follow.

Claim 1. $\left|H:\left(H \cap H^{a}\right)\right|=2 r$, the core of $H \cap H^{a}$ in $H$ is $\operatorname{Alt}\left(X_{1}\right) \times \cdots \times \operatorname{Alt}\left(X_{m-2}\right) \times$ $\operatorname{Alt}\left(X_{m}\right)$ and the action of $H$ on the right cosets of $H \cap H^{a}$ is equivalent to the imprimitive action of $\operatorname{Alt}(r)$ wr $C_{2}$ of degree $2 r$.

Set $K=\operatorname{Alt}\left(X_{0}^{\prime}\right) \times \operatorname{Alt}\left(X_{1}\right) \times \cdots \times \operatorname{Alt}\left(X_{m-1}\right) \times \operatorname{Alt}\left(X_{m}\right)$. Note that $K$ is the stabiliser in $H$ of the point $r$ of $\Omega$. As a normalises $K$, we have $K \subseteq H \cap H^{a}$. The orbits of $r$ under $H$ and $H^{a}$ are

$$
r^{H}=\{1, \ldots, r, 1+(m-1) r, \ldots, r+(m-1) r\}=X_{0} \cup X_{m-1}
$$

and

$$
\begin{aligned}
r^{H^{a}} & =\left(r^{a}\right)^{H a}=r^{H a}=\{1, \ldots, r, 1+(m-1) r, \ldots, r+(m-1) r\}^{a} \\
& =\{1+m r, \ldots, r-1+m r, r, 1+(m-2) r, \ldots, r+(m-2) r\} \\
& =\{r\} \cup X_{m-2} \cup X_{m} .
\end{aligned}
$$

Let $g \in H \cap H^{a}$. We have $r^{g} \in r^{H} \cap r^{H^{a}}=\{r\}$, and hence $g$ fixes $r$. Therefore $H \cap H^{a} \subseteq K$. This yields $K=H \cap H^{a}$ and $\left|H:\left(H \cap H^{a}\right)\right|=2 r$.

Finally, as $H \cap H^{a}$ is the stabiliser in $H$ of the point $r$, we have that the core of $H \cap H^{a}$ in $H$ is the point-wise stabiliser of the set $r^{H}=X_{0} \cup X_{m-1}$, which is clearly $\operatorname{Alt}\left(X_{1}\right) \times \cdots \times \operatorname{Alt}\left(X_{m-2}\right) \times \operatorname{Alt}\left(X_{m}\right)$. 
It is easy to compute that

$$
h a:\left\{\begin{array}{llll}
z & \mapsto z+(m-2) r & & \text { for } 1 \leq z \leq r, \\
z+l r & \mapsto z+(m-l) r & & \text { for } 1 \leq z \leq r, 1 \leq l \leq m-1, l \text { odd, } \\
z+l r & \mapsto z+(m-2-l) r & & \text { for } 1 \leq z \leq r, 1 \leq l \leq m-3, l \text { even, } \\
z+(m-1) r & \mapsto z+m r & & \text { for } 1 \leq z \leq r-1, \\
r+(m-1) r & \mapsto r, & & \text { for } 1 \leq z \leq r-1 . \\
z+m r & \mapsto z &
\end{array}\right.
$$

Using this equation for the permutation $h a$, we obtain that $\langle h a\rangle$ has $r$ orbits. Specifically, for each $z \in\{1, \ldots, r-1\}$, the set $\{z+j r \mid j=0, \ldots, m\}$ is an orbit of $\langle h a\rangle$ of size $m+1$. Also, $\{r+j r \mid j=0, \ldots, m-1\}$ is an orbit of $\langle h a\rangle$ of size $m$.

Claim 2. $\operatorname{Alt}(\Omega) \subseteq\langle H, a\rangle$.

Set $L=\langle H, a\rangle, g=(h a)^{m+1}$ and $T=\langle H, g\rangle$. Since for each $j \in\{0, \ldots, m-1\}$ the group $H$ is transitive on $X_{j}$ and $g$ is a cycle with support $\{r, 2 r, \ldots, m r\}$, we obtain that $T$ is transitive on $\{1, \ldots, m r\}=\Omega \backslash X_{m}$ and fixes point-wise $X_{m}$. Since $T \subseteq L, X_{0}^{\prime a}=X_{m}$ and $a \in L$, we obtain that $L$ is transitive on $\Omega$.

As $T$ is transitive on $\Omega \backslash X_{m}$ and fixes point-wise $X_{m}$, using the definition of $a$, we see that $S=T^{a}$ acts transitively on $\Omega \backslash X_{0}^{\prime}$ and fixes point-wise $X_{0}^{\prime}$. For each $i \in\{2, \ldots, r\}$, fix $x_{i}$ an element of $\operatorname{Alt}\left(X_{0}\right) \subseteq H$ with $r^{x_{i}}=i$. Clearly, $S^{x_{i}}$ fixes point-wise $\{1, \ldots, i-1, i+1, \ldots, r\}$ and acts transitively on $\{i, r+1, \ldots, r-1+m r\}$. Therefore $\left\langle S^{x_{i}} \mid i=2, \ldots, r\right\rangle \subseteq L$ is transitive on $\{2, l+1, \ldots, r-1+m r\}=$ $\Omega \backslash\{1\}$. This shows that $L$ is 2 -transitive. Since $L$ contains a 3 -cycle, we obtain that $\operatorname{Alt}(\Omega) \subseteq L$.

Claim 3. $G=\langle H, a\rangle$.

The permutation $h$ is an involution fixing point-wise $X_{(m-1) / 2} \cup X_{m}$. Therefore $h$ has $(m-1) r / 2$ cycles. If $r$ is odd, then (as $m \equiv 3 \bmod 4)$ we obtain $h \notin$ $\operatorname{Alt}(\Omega)$ and hence (from Claim 2) $G=\langle H, a\rangle$. We may thus assume that $r$ is even. The permutation $a$ is an involution fixing only the point $r$. Therefore $a$ has $(r-2+m r) / 2=(m-1) r / 2+(r-1)$ cycles. Since $m \equiv 3 \bmod 4$ and $r$ is even, we have $a \notin \operatorname{Alt}(\Omega)$, and hence $G=\langle H, a\rangle$.

As $a$ is an involution, from Claim 3 we have that $\Gamma$ is a connected $G$-arc-transitive graph. Now Claim 1 gives that $\Gamma$ has valency $2 r$ and that, for a vertex $v, G_{v}^{\Gamma(v)} \cong$ $\operatorname{Alt}(r)$ wr $C_{2}$ (in its imprimitive action of degree $2 r$ ) and $G_{1}(v)=\operatorname{Alt}\left(X_{1}\right) \times \cdots \times$ $\operatorname{Alt}\left(X_{m-2}\right) \times \operatorname{Alt}\left(X_{m}\right) \cong \operatorname{Alt}(r)^{m-2} \times \operatorname{Alt}(r-1)$.

Now consider the generating set $H a H$ of $\operatorname{Sym}(\Omega)$. It has size $2 r|H|$, and, as in the proof of Lemma 15, we see that $\left|(H a H)^{3}\right| \leq(2 r)^{3}|H|=(2 r)^{2}|H a H|$.

Remark 18. A more elaborate and general version of Theorem 17 is in [20]. Indeed, for any composite positive integer $r s$ (where $r>1, s>1$ ) and any transitive permutation groups $R$ of degree $r$ and $S$ of degree $s, 20$, Theorem 2] gives an infinite family of graphs $\Gamma_{m}$ (where $m \geq r s$ is odd) of valency $r s$ admitting a $G_{m}$-arc-transitive action (where $G_{m}=\operatorname{Alt}(r s m+r-1)$ or $\operatorname{Sym}(r s m+r-1)$ ) such that a vertex stabiliser in $G_{m}$ induces $R$ wr $S$ (in its imprimitive action) on the neighbourhood of the vertex and with kernel $R^{m-2} \times R_{1}$ (where $R_{1}$ is a point stabiliser in $R$ ). 


\section{REFERENCES}

[1] L. Babai, P. J. Cameron, P. P. Pálfy, On the orders of primitive groups with restricted nonabelian composition factors, J. Algebra 79 (1982), 161-168. MR679977(84e:20003)

[2] J. Bourgain, A. Gamburd and P. Sarnak, Affine linear sieve, expanders and sum product, Inventiones Math. 179 (1) (2010), 559-644. MR2587341 (2011d:11018)

[3] J. Bourgain and P. P. Varjú, Expansion on $S L_{d}(Z / q Z), q$ arbitrary, to appear in Inventiones Math., DOI:10.1007/x00222-011-0345-4Online First ${ }^{\mathrm{TM}}$.

[4] E. Breuillard, B. Green, T. Tao, Approximate subgroups of linear groups, Geometric and Functional Analysis 21, no. 4, 774-819, DOI:10.1007/s00039-011-0122-y.

[5] E. Breuillard, B. J. Green and T. C. Tao, Suzuki groups as expanders, Groups Geom. Dyn. 5 (2011), no. 2, 281-299. MR2782174

[6] E. Breuillard, B. J. Green, R. Guralnick and T. C. Tao, Expansion in finite simple groups of Lie type, in preparation.

[7] W. Bosma, J. Cannon, C. Playoust, The Magma algebra system. I. The user language, J. Symbolic Comput. 24 (1997), 235-265. MR1484478

[8] P. J. Cameron, C. E. Praeger, J. Saxl, G. M. Seitz, On the Sims conjecture and distance transitive graphs, Bull. Lond. Math. Soc. 15 (1983), 499-506. MR705530 (85g:20006)

[9] M. D. Conder, C. H. Li, C. E. Praeger, On the Weiss conjecture for finite locally primitive graphs, Proc. Edinburgh Math. Soc. 43 (2000), 129-138. MR1744704 (2001e:05054)

[10] A. A. Ivanov and S. V. Shpectorov, Amalgams determined by locally projective actions, Nagoya Math. J. 176 (2004), 19-98. MR2108123 (2005m:20057)

[11] W. M. Kantor, E. M. Luks, Computing in quotient groups, STOC '90 Proceedings of the twenty-second ACM symposium on theory of computing (1990), 524-534.

[12] A. Lubotzky, D. Segal, Subgroup growth, Birkhäuser, 2003. MR.1978431 (2004k:20055)

[13] C. E. Praeger, Imprimitive symmetric graphs, Ars Combinatoria 19A (1985), 149-163. MR790928 (86k:05058)

[14] C. E. Praeger, Finite quasiprimitive graphs, in Surveys in combinatorics, London Mathematical Society Lecture Note Series, vol. 24 (1997), 65-85. MR1477745 (99b:05076)

[15] C. E. Praeger, Finite transitive permutation groups and bipartite vertex-transitive graphs, Illinois Journal of Mathematics 47 (2003), 461-475. MR2031334(2005d:20004)

[16] C. E. Praeger, P. Spiga and G. Verret, Bounding the size of the vertex-stabiliser in vertextransitive graphs, preprint: arXiv:1102.1543.

[17] L. Pyber, E. Szabó, Growth in finite simple groups of Lie type of bounded rank, preprint: arXiv: 1005.1858

[18] G. Sabidussi, Vertex-transitive graphs. Monatsh. Math. 68 (1964), 426-438. MR0175815 $(31: 91)$

[19] C. C. Sims, Graphs and finite permutation groups, Math. Z. 95 (1967), 76-86. MR0204509 $(34: 4348)$

[20] P. Spiga, Two local conditions on the vertex stabiliser of arc-transitive graphs and their effect on the Sylow subgroups, to appear in Journal of Group Theory, DOI:10.1515/JGT.2011.097.

[21] J. G. Thompson, Bounds for the orders of maximal subgroups, J. Algebra 14 (1970), 135-138. MR.0252500 (40:5720)

[22] V. I. Trofimov, Graphs with projective suborbits. Exceptional cases of characteristic 2. IV, Izv. Ross. Akad. Nauk Ser. Mat. 67 (2003), 193-222; translation in Izv. Math. 67 (2003), 1267-1294. MR2032095 (2005a:05110)

[23] V. I. Trofimov and R. M. Weiss, The group $E_{6}(q)$ and graphs with a locally linear group of automorphisms, Math. Proc. Cambridge Philos. Soc. 148 (2010), 1-32. MR 2575369 (2011b:20006)

[24] R. Weiss, s-transitive graphs, Colloq. Math. Soc. János Bolyai 25 (1978), 827-847. MR642075 (83b:05071)

[25] R. Weiss, Symmetric graphs with projective subconstituents, Proc. Amer. Math. Soc. 72 (1978), 213-217. MR524349 (80h:05031)

[26] R. Weiss, Symmetrische Graphen mit auflsbaren Stabilisatoren (German), J. Algebra 53 (1978), 412-415. MR502640(80a:05114)

[27] R. Weiss, An application of $p$-factorization methods to symmetric graphs, Math. Proc. Cambridge Philos. Soc. 85 (1979), 43-48. MR510398 (81b:05059) 
Centre for Mathematics of Symmetry and Computation, School of Mathematics and Statistics, The University of Western Australia, Crawley, WA 6009, Australia

E-mail address: praeger@maths.uwa.edu.au

Rényi Institute of Mathematics, Hungarian Academy of Sciences, P. O. Box 127, H-1364 Budapest, Hungary

E-mail address: pyber@renyi.hu

Centre for Mathematics of Symmetry and Computation, School of Mathematics and Statistics, The University of Western Australia, Crawley, WA 6009, Australia

E-mail address: spiga@maths.uwa.edu.au

Rényi Institute of Mathematics, Hungarian Academy of Sciences, P. O. Box 127, H-1364 Budapest, Hungary

E-mail address: endre@renyi.hu 